University of New Mexico

UNM Digital Repository

Undergraduate Medical Student Research

Health Sciences Center Student Scholarship

6-30-1905

\title{
Effect of Carelink, an internet-based insulin pump monitoring system, on glycemic control in rural and urban children with type 1 diabetes mellitus
}

Erin Corriveau

Peter Durso

Ellen Kaufman

Betty Skipper

Louise Laskaratos

See next page for additional authors

Follow this and additional works at: https://digitalrepository.unm.edu/ume-research-papers

\section{Recommended Citation}

Corriveau, Erin; Peter Durso; Ellen Kaufman; Betty Skipper; Louise Laskaratos; and Kimberly Heintzman. "Effect of Carelink, an internet-based insulin pump monitoring system, on glycemic control in rural and urban children with type 1 diabetes mellitus."

(1905). https://digitalrepository.unm.edu/ume-research-papers/54

This Article is brought to you for free and open access by the Health Sciences Center Student Scholarship at UNM Digital Repository. It has been accepted for inclusion in Undergraduate Medical Student Research by an authorized administrator of UNM Digital Repository. For more information, please contact disc@unm.edu. 


\section{Authors}

Erin Corriveau, Peter Durso, Ellen Kaufman, Betty Skipper, Louise Laskaratos, and Kimberly Heintzman 


\section{Original Article}

\section{Effect of Carelink, an internet-based insulin pump monitoring system, on glycemic control in rural and urban children with type 1 diabetes mellitus}

Corriveau EA, Durso PJ, Kaufman ED, Skipper BJ, Laskaratos LA, Heintzman KB. Effect of Carelink, an internet-based insulin pump monitoring system, on glycemic control in rural and urban children with type 1 diabetes mellitus.

Pediatric Diabetes 2008: 9(Part II): 360-366.

Objective: To determine whether use of the internet-based insulin pump monitoring system, Carelink, improved glycemic control in rural and urban children treated with insulin pump therapy.

Research design: We reviewed records of 94 children treated with insulin pump therapy between the years 2004 and 2007 and compared glycemic control, diabetes self-care measures, frequency of clinic visits, and geographic location associated with Carelink use.

Results: Carelink users showed improvement in hemoglobin Alc (HbAlc) levels $[8.0 \pm 0.1$ (SE) vs. $7.7 \pm 0.1$ (SE), $p=0.002]$. Carelink users uploaded pump and glucometer data $2.2 \pm 1.8$ (SD) times per month over $0.8 \pm 0.4$ (SD) yr. Patients who had no access to CARELINK software and were followed in a conventional manner showed no change in HbAlc levels [8.0 00.2 (SE) vs, $8.1 \pm 0.2$ (SE), $p=0.17$ ] during the study period. Carelink non-users, defined as patients who had Carelink access but did not use it, had a higher HbAlc level at the start of the study and did not change over the study period $[8.9 \pm 0.2$ (SE) vs. $9.0 \pm 0.3(\mathrm{SE}), \mathrm{p}=0.82$ ]. Rural Carelink users showed improvement in HbAlc levels following Carelink use $[7.9 \pm 0.2$ (SE) vs. $7.4 \pm 0.2$ (SE), $\mathrm{p}=0.001]$, yet had significantly fewer clinic visits per year compared with urban patients $[2.8 \pm 0.2$ (SE) ys. $3.5 \pm 0.1$ (SE), $\mathrm{p}=0.001]$.

Conclusion: Use of the Carelink system was associated with improved glycemic control in children with type 1 diabetes on insulin pump therapy.

\author{
Erin A Corriveau ${ }^{a}$, \\ Peter J Durso ${ }^{a}$, \\ Ellen D Kaufman', \\ Betty J Skipperc, \\ Louise A Laskaratos ${ }^{b}$ and \\ Kimberly B Heintzman ${ }^{\text {b }}$ \\ aUniversity of New Mexico School of \\ Medicine, Albuquerque, NM, USA; \\ bresbyterian Medical Group; and \\ cDepartment of Family and Community \\ Medicine, University of New Mexico \\ School of Medicine, Albuquerque, NM, \\ USA
}

Key words: Carelink internet-based insulin pump monitoring system children with type 1 diabetes - insulin pump therapy - internet-based data analysis

Corresponding author:

Ellen D Kaufman, MD

201 Cedar SE

Suite 4640

Albuquerque

NM 87106

USA.

Tel: 505-563-6530;

fax: 505-563-6325;

e-mail: ekaufman@phs.org

Submitted 14 August 2007. Accepted for publication 8 November 2007
The incidence of childhood type 1 diabetes has increased worldwide (1) and in the USA (2). At the same time, more stringent blood glucose targets have been recommended for children $(3,4)$ with the longterm goal of preventing diabetes-related complications (5-7). There has been an increased interest in insulin pump therapy as a means of achieving these goals in multiple pediatric age-groups (8-14). However, the success of this therapy has been linked to increased contact between diabetes care providers and patients in order to make the frequent dose adjustments that growing children require for optimal blood glucose control (15). The increasing clinical burden of childhood diabetes is occurring at a time when skilled pediatric endocrinologists and nurse practitioners are scarce. With more than 200.000 children in the USA 
affected by diabetes, there are only 633 full-time clinical pediatric endocrinologists. Most of these practitioners are located in university centers in major cities, with only $3 \%$ of practitioners in rural areas (16). In New Mexico, the fifth largest state in which $60 \%$ of the population lives in rural areas, there are only two centers of pediatric diabetes care, both located in Albuquerque. The goal of visits to the diabetes clinic every 3 months is not possible for many patients because of their distance from clinic. This paper examines the use of the internet-based insulin pump monitoring system, Carelink, to determine if evaluation of insulin pump and blood glucose data through the internet, by both parents and diabetes care providers, can improve glycemic control in children with type 1 diabetes.

\section{Patient population and methods}

Data were collected from record reviews of 94 children and adolescents with type 1 diabetes mellitus. All patients on insulin pump therapy who had available data before and after Carelink use between 2004 and 2007 were included in this study. Exempt status based on record review was obtained from the Presbyterian Medical Group Human Research Review Committee. Each patient was educated by a Certified Diabetes Educator (CDE), nurse pump trainer (L. A. L.) and a CDE registered dietician (K. B. H.). The diabetes education program and the pediatric endocrinologist in this community hospital-based clinic are recognized by the American Diabetes Association and National Committee for Quality Assurance. Patients were seen at 3-month to 1-yr intervals based on their geographic proximity to our clinic in Albuquerque, NM, USA. Rural patients were defined as living $1 \mathrm{~h}$ or more away from our clinic. Thirty-seven percent of the patients studied lived in a rural location (Fig. 1). At each visit, the patient was examined, glucometer and insulin pump data were uploaded and reviewed, and a fingerstick hemoglobin Alc ( $\mathrm{HbAlc}$ ) was performed using the DCA 2000 (Bayer, Tarrytown, NY).

The Medtronic insulin pump was used by 92 of 94 patients. Prior to 2005, the Medtronic solutions software was used at clinic visits to upload pump data for review. Patients did not have access to this program for home use. When the Medtronic CARELINK software became available in 2005, all patients using the Medtronic 515, 522, 715, and 722 pumps were upgraded to the Carelink system. One patient used a Cozmo insulin pump, and one patient used an Animas insulin pump. COZMo and ANIMAS software were used at clinic visits to obtain pump and glucometer data in these patients. However, Cozmo and Animas patients could not upload their pump data from home. Patients using Cozmo, Animas, and older model Medtronic pumps did not have access to Carelink.

\section{CARELINK Software}

We began using the CARELINK software program provided by the Medtronic company in January 2005. This software was provided free of charge by Medtronic to our clinic and to each patient who used a compatible Medtronic insulin pump, along with a universal serial bus (USB) cable for use with a home computer. Patients were assigned user IDs and passwords of their choice and were taught how to upload their insulin pump and meter to the Medtronic Carelink internet site. For those patients who did not have access to a home computer, the system was set up at their primary care physicans' office or at the home of a relative. The patients and parents were instructed by E. D. K. or L. A. L. at clinic visits how to review the Carelink reports in order to evaluate their blood sugar levels and insulin pump settings as follows:

(i) Quick view 14 day summary - To view the average daily blood sugar level and glucose excursions, the number of tests per day, the units of insulin used per day, \% bolus to basal insulin, the number of boluses per day, and the grams of carbohydrate entered into the pump per day;

(ii) Modal day report - To view a graphic representation of when blood sugars were in or out of goal range based on time of day, and to view premeal, postmeal, and overnight blood sugar averages;

(iii) Daily logbook or data table - To review the specifics of each day. Data in this report were organized so that parents and providers could view when insulin boluses for meals were omitted or when there appeared to be a pump or catheter malfunction;

(iv) Daily summary report - To determine when blood sugar levels were performed on the Becton Dickinson link meter or when they were entered manually into the pump; and

(v) Pump or device settings - To view the current basal rates, carbohydrate ratios, sensitivity (correction) factors, goal blood sugar ranges, and assigned duration of insulin action (insulin on board).

Patients were instructed to e-mail their diabetes care provider (E. D. K. or L. A. L.) once an upload was performed. With the patients' permission, the diabetes care provider could then sign on to the web site and view the data. Patients were asked to upload weekly to monthly based on their clinical status. Patients who failed to upload received several reminder phone calls, 


\section{Corriveau et al.}

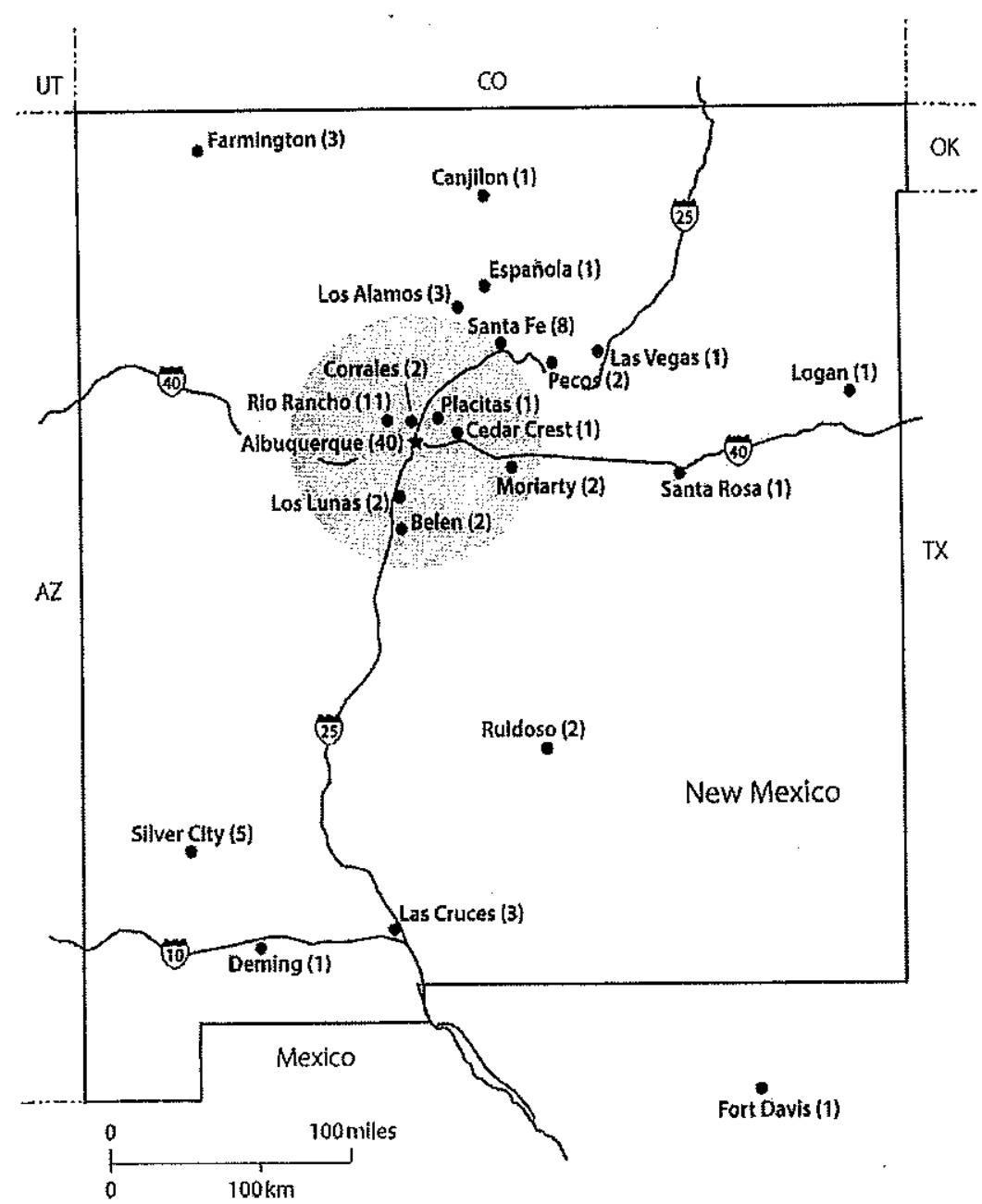

Fig. 1. Geographic location of pump patients (total number of patients $=94$ ). $\star$, pediatric endocrine clinics. The shaded region indicates areas within $1 \mathrm{~h}$ of Albuquerque.

and a reminder e-mail was sent to the parents as well. The great majority of uploads were done by the parents. In some cases, teenagers did the uploads, but replies were always sent to the child and the parent. Some patients were able to make their own dose adjustments, and others waited for the diabetes care provider to contact them through e-mail with recommendations. In cases where an urgent change appeared necessary, the parent was also contacted by phone. The Carelink program provided summary reports only and did not create therapeutic recommendations. An average of 10 min was spent by the provider to review one patient's report and reply through e-mail to the patient. Sample Carelink reports can be viewed at http:// hsc.unm.edu/som/fcm/CarelinkPersonalReports.pdf.

The 94 patients were divided into three groups:

'No-access' group consisted of patients without Carelink access because their pumps were not compatible with CARELINK software ( 33 patients).
'Non-users' group consisted of patients who had access to CARELINK software but did not use it (20 patients).

'Carelink users' group consisted of patients who used the Carelink system to upload and review their pump and glucometer data (41 patients).

Of the total of 94 patients, 61 had Carelink access and 33 patients had no access.

\section{Data collection}

Recorded variables consisted of patient age, duration of diabetes before start of pump therapy, duration of pump therapy, HbAlc, Carelink uploads per month, frequency of day and night blood sugar testing, selfadjustment of insulin doses, frequency of clinic visits, insurance type, and geographic location. The duration of pump therapy was defined as the time between starting pump therapy to the last visit in the study. 
Data were analyzed pre- and post-Carelink use. Study eligibility required that a patient have at least one $\mathrm{HbAl} c$ value in the 'pre' period and at least one $\mathrm{HbAlc}$ value in the 'post' period. The time periods were defined as follows

\section{Pre-Carelink use}

Carelink users and non-users: 'Pre' period started 1 yr before the registration and instruction in Carelink use and ended $14 \mathrm{~d}$ after the initial Carelink instruction. We defined the 'pre-Carelink' period to include $14 \mathrm{~d}$ after Carelink instruction. This was done to make sure that the HbAlc done on the day of Carelink instruction was included in the 'pre-Carelink' period and because patients were instructed to do their first upload 2 wks after the initial instruction date.

No-access patients: The index date assigned was 1 January 2005 when Carelink became available in our clinic. The 'Pre' period started on 1 January 2004 and ended on 15 January 2005.

Post-Carelink use

Carelink users and non-users: The 'post' period started $15 \mathrm{~d}$ after beginning the use of the Carelink system and ended at the end of the data collection period on 28 February 2007.

No-access patients: The 'post' period started on 16 January 2005 and ended on 28 February 2007.

\section{Statistical analysis}

Data were abstracted onto worksheets by the pediatric endocrinologist, entered into a Microsoft ACCESS database, and transferred to a sas data set using STATTRANSFER software. Baseline clinical characteristics were compared among the three groups using chisquared or Fisher's exact tests for categorical variables, one-way analysis of variance for normally distributed continuous variables (age and duration of access to Carelink), and the Kruskal-Wallis test for nonnormally distributed variables (duration of diabetes, duration of pump therapy, and time using continuous subcutaneous insulin infusion (CSII) before the start of Carelink for the non-user and user groups or the index date for the no-access group). For each patient, mean $\mathrm{HbAlc}$ level values were calculated for the year before initiating Carelink and the time period following Carelink initiation as defined above. Paired $t$-tests were used to compare changes in mean $\mathrm{HbAlc}$ levels before and after initiation of CARELINK software within each group. One-way analysis of variance was used to compare mean HbAlc levels among groups. Fisher's exact tests were used to compare HbAlc categories among'groups. Two-way analysis of variance was used to test annualized visit rates between patients residing in urban and rural areas adjusted for study group. McNemar's test was used to compare percentages of
Carelink users with $\mathrm{HbAlc}$ values $\leq 7.5 \%$ before and after using Carelink. All calculations were done using $\mathrm{SAS}^{\text {B }}$ software version 9.1. An additional analysis was conducted using the generalized estimating equation approach for longitudinal data with the $\mathrm{HbAlc}$ value as a continuous variable and age as a covariate. The results (data not shown) were unchanged from analyses listed above.

\section{Results}

Characteristics of the three groups are summarized in Table 1.

Analysis of $\mathrm{HbAlc}$ data before and after initiation of Carelink use is summarized in Table 2 and is demonstrated in Fig. 2.

Rural Carelink users showed improvement in $\mathrm{HbA1c}$ levels after Carelink use [7.9 \pm 0.2 (SE) vs. $7.4 \pm 0.2$ (SE), $\mathrm{p}=0.001]$, whereas rural non-users $[9.2 \pm 0.5$ (SE) vs. $9.2 \pm 0.5$ (SE), $\mathrm{p}=0.96$ ] and rural no-access patients $[8.1 \pm 0.3$ (SE) vs. $8.0 \pm 0.4(\mathrm{SE}), \mathrm{p}=0.79]$ did not change. There were no statistically significant changes in HbAlc before and after Carelink use in the groups of urban patients. Rural patients had significantly fewer annualized clinic visits $[2.8 \pm 0.2$ (SE) vs. $3.5 \pm 0.1$ (SE) for urban patients, $p=0.003$ ]. Rural Carelink users uploaded $2.3 \pm 0.5$ (SE) times per month vs. urban Carelink users who uploaded $2.1 \pm$ 0.3 (SE) times per month, $\mathrm{p}=0.75$.

No-access patients and Carelink users had similar diabetes self-care behaviors. They did not differ significantly in frequency of adjusting doses (50 vs. $68 \%$ ), testing overnight ( 50 vs. $64 \%$ ), or tests per day. Non-users did differ significantly from the other groups in adjusting insulin doses $(23 \%), p=0.03$, and testing overnight $(15 \%), p=0.01$, but not in frequency of blood glucose monitoring. The mean frequency of blood glucose monitoring per day was $5.4 \pm 2.3$ (SD) for no-access patients, $4.3 \pm 1.9$ (SD) for non-users, and $5.3 \pm 1.5(\mathrm{SD})$ for Carelink users, $\mathrm{p}=0.13$.

\section{Discussion}

Rural patients who used the Carelink system to upload and analyze their insulin pump and blood glucose data showed significant improvement in glycemic control. We believe that this was because of increased patient contact with medical staff through the internet and access for parents and care providers to more accurate blood glucose, insulin administration, and carbohydrate gram data.

Pankowska et al. (15) suggest in a study of 100 children treated with insulin pump therapy that the intensity of supervision is reflected in the level of metabolic control, with more frequent visits resulting in a better outcome. This led to their recommendation 


\section{Corriveau et al.}

Table 1. Clinical characteristics of insulin pump patients

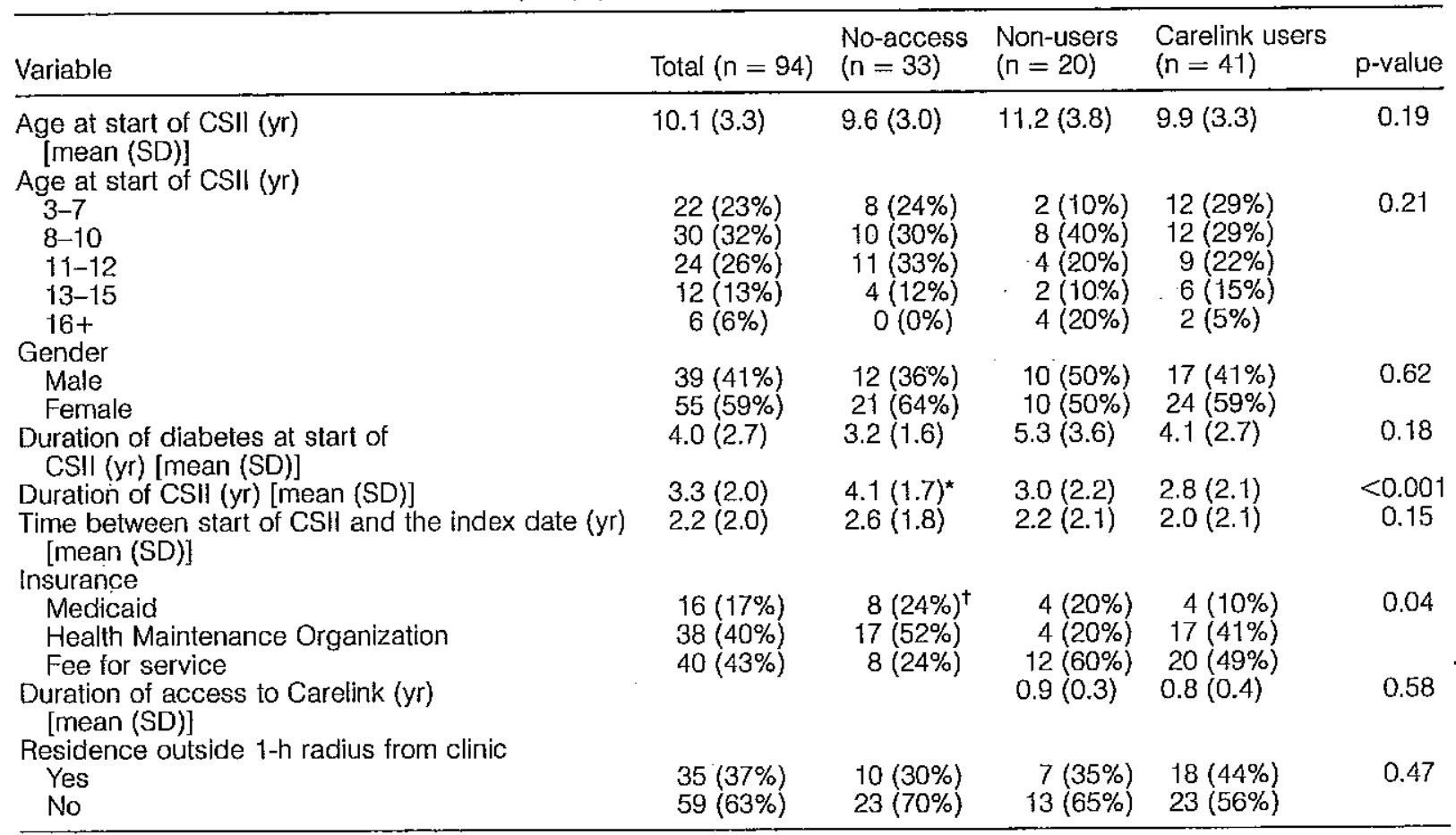

CSII, continuous subcutaneous insulin infusion.

*The mean for the no-access group is significantly different from the means for the other groups.

tThe distribution for the no-access group is significantly different from the distribution for the non-user.

that children be seen every 2 months. Cho et al. (17) reported significantly improved glycemic control in 120 patients who used an internet-based monitoring system to communicate with medical staff, who made 1586 recommendations over 30 months. Of these recommendations, $41.7 \%$ were as 'encouragement', $33.5 \%$ were as 'problem assessment and counseling', and only $12 \%$ involved drug modification. Patients who used this internet-based system had significantly better glycemic control compared with conventionally treated patients.

In our practice, 41 Carelink users uploaded an average of 2.2 times per month over a 10 -month period of time, resulting in 902 interactions with their care providers. Children from rural areas benefited equally compared with urban children, even though they had significantly fewer clinic visits.
No-access patients and Carelink users had similar self-care behaviors. They tested as frequently, adjusted insulin doses, and monitored blood sugar overnight with the same frequency. No-access patients had identical HbAlc levels compared with Carelink users in the pre-Carelink period. We feel that the significant improvement in HbAlc levels in Carelink users was because of increased contact through the internet with their diabetes care providers and increased access to their childs' pump data by parents. This ensured that two people, the parent and the care provider, were able to analyze the childs' blood glucose and insulin data to look for patterns, indicating the need for a dose change or a potential problem such as missed boluses. If no problems were evident, the parents benefited from the encouragement that they received for a job well done.

Table 2. Analysis of hemoglobin Aic data before and after initiation of Carelink use

\begin{tabular}{lllllcc}
\hline Group & $\begin{array}{l}\text { Uploads/month } \\
\text { [mean (SD)] }\end{array}$ & $\mathrm{n}$ & $\begin{array}{l}\text { Before } \\
\text { [mean (SE)] }\end{array}$ & $\begin{array}{l}\text { After } \\
\text { [mean (SE)] }\end{array}$ & $\begin{array}{l}\text { Difference } \\
\text { [mean (SE)] }\end{array}$ & $\mathrm{p}$-value \\
\hline No-access & N/A & 33 & $8.0(0.2)$ & $8.1(0.2)$ & $0.2(0.1)$ & 0.17 \\
Non-users & N/A & 20 & $8.9(0.2)^{\star}$ & $9.0(0.3)^{\star}$ & $0.1(0.2)$ & 0.82 \\
Carelink users & $2.2(1.8)$ & 41 & $8.0(0.1)$ & $7.7(0.1)$ & $-0.4(0.1) \dagger$ & 0.002 \\
p-value & & & 0.002 & $<0.001$ & 0.01 & \\
\hline
\end{tabular}

N/A, not applicable.

*The mean for the non-user group is significantly different from the means for the other groups.

tThe mean for the user group is significantly different from the means for the other groups. 
Effect of Carelink on glycemic control
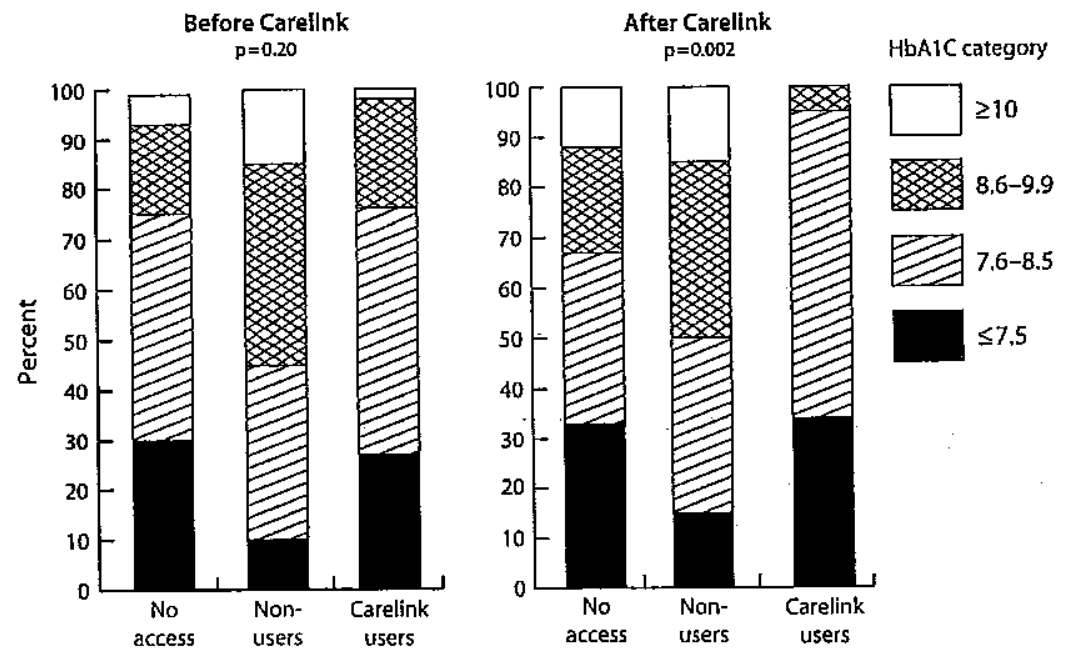

Fig. 2. Hemoglobin Alc (HbAlc) category vs. Carelink status. No access: patients without Carelink access; Non-users: patients with Carelink access who did not use it; Carelink users: patients who used Carelink.

We are not sure why the rural users showed the major benefit. It is possible that this group of parents were more highly motivated as they had to make a much greater effort to be seen at a pediatric diabetes center rather than obtain care from primary care providers in their own community.

Non-users, who were given access to Carelink but did not use it, were significantly different from the other two groups in level of glycemic control at the start and end of the study period. This group could be described as non-adherent; yet, they tested their blood sugar a mean of 4.3 times per day. However, they did not react to persistently elevated blood sugars by changing their diabetes care plan, and we did not see them frequently enough to adequately perform this function for them. It is important to note that this group had a higher percentage of adolescent patients in whom compliance is known to be more difficult to achieve (12-14).

Carelink users, their parents, and diabetes care providers had the additional benefit of an organized software program to view and analyze data. Children do best when their parents and physicians have accurate records that are critical to identify problems and adjust doses. In the past, the ability to make these adjustments has been limited, even for providers, by the lack of accurate patient data. The advantage of the Carelink system is that an accurate daily log of blood sugars, insulin doses, and carbohydrate grams is available to the diabetes care provider, the parent, and the child. The challenge that remains is to convince parents, patients, and providers to view these data on a regular basis so that they can be used to adjust and evaluate therapy. In our experience, it takes less time to view Carelink reports than to interpret faxed log sheets or blood sugars that have been called in and recorded by hand. As evidenced by the current study, this is especially valuable for patients who live far from clinic and cannot be seen frequently.

The disappointment to us in this study is that although there was an improvement in glycemic control after Carelink use, the percentage of patients with a $\mathrm{HbA} 1 \mathrm{c}$ of $7.5 \%$ or less only increased from 27 to $37 \%$. The gains in glycemic control were not large, and although $95 \%$ of Carelink users had HbA1c levels of $<8.5 \%$, this is far from ideal control. We plan to address this issue in future research by creating a tool for interpreting Carelink reports that parents and providers can use in order to make more appropriate and consistent dose adjustments and by studying this in a prospective manner. We feel that part of the problem in our educational program is insufficient emphasis on the need for parents to look at the reports to determine when change is needed vs. learning the technical aspects of pump use. We also hope to improve the effectiveness of the Carelink system with the use of the pumpaugmented continuous glucose monitoring system, which we began to use in our practice in June 2007.

Successful pediatric diabetes programs have been described as a cooperative venture between committed families and a multidisciplinary diabetes team (18). Carelink technology is a useful tool in achieving this goal.

\section{Acknowledgement}

We gratefully acknowledge the support for this project that was provided by Bill and Marge Mandly and the Presbyterian Healthcare Foundation.

\section{References}

1. Gale EA. The rise of childhood type 1 diabetes in the 20th century. Diabetes 2002: 51: 3353-3361. 


\section{Corriveau et al.}

2. VehiK $\mathrm{K}$, Hamman $\mathrm{R}$, Lezotte $\mathrm{D}$ et al. Increasing incidence of type 1 diabetes in 0-17-year-old Colorado youth. Diabetes Care 2007: 30: 503-509.

3. WILLI SM. How low can we go...safely? Factors affecting intensive diabetes management. J Pediatr 2006: 149: 227-232.

4. American Diabetes Association, Standards of medical care in diabetes. Diabetes Care 2005: 28 (Suppl. 1): \$4S36.

5. The DCCT Research Group. The effect of intensive treatment of diabetes on the development and progression of long-term complications in insulin-dependent diabetes mellitus. The Diabetes Control and Complications Trial. N Engl J Med 1993: 329: 977-986.

6. Schultz CJ, Konepelska-Bahu T, Dalton RN et al. Microalbuminuria prevalence varies with age, sex and puberty in children with type 1 diabetes followed from diagnosis in a longitudinal study. Oxford Regional Prospective Study Group. Diabetes Care 22 1999: 495502.

7. Donaghue KC, Fung AT, Hing $S$ et al. The effect of prepubertal diabetes duration on diabetes: microvascular complications in early and late adolescence. Diabetes Care 1997: 20: 77-80.

8. LitTon J, Rice A, Friedman O, Lee M, Fregmark M. Insulin pump therapy in toddlers and preschool children with type 1 diabetes mellitus. J Pediatr 2002: 141: 490-495.

9. Weinzimer S, Ahern J, Doyle E et al. Persistence of benefits of continuous subcutaneous insulin infusion in very young children with type 1 diabetes: a follow-up report. Pediatrics 2004: 114: 1601-1605.

10. Dimeglio L, Pottorff T, Boyd S, France L, Fineberg N, Eugster E. A randomized controlled study of insulin pump therapy in diabetic preschoolers. J Pediatr 2004: 145: $380-384$.

11. Weinzimer S, Swan K, Sikes K, Ahern J. Emerging evidence for the use of insulin pump therapy in infants, toddlers and preschool-aged children with type 1 diabetes. Pediatr Diabetes 2006: 7 (Suppl. 4): 15-19.

12. Kordonouri O, Hartmann R, Lauterborn R, BarneKow C, HobFFe J, DeIss D. Age-specific advantages of continuous subcutaneous insulin infusion as compared with multiple daily injections in pediatric patients. Diabetes Care 2006: 29: 133-134.

13. Willi S, Planton J, Egede L, Schwarz S. Benefits of continuous subcutaneous insulin infusion in children with type 1 diabetes. J Pediatr 2003: 143: 796-801.

14. BatTELino $T$. Risk and benefits of continuous subcutaneous insulin infusion treatment in school children and adolescents. Pediatr Diabetes 2006: 7 (Suppl. 4): 2024.

15. Pankowska E, Slorka A, SzpowsKa A, LipKa $M$. Memory of insulin pumps and their record as a source of information about insulin therapy in children and adolescents with type 1 diabetes. Diabetes Technol Ther 2005: 7: 308-314.

16. Althouse L, Stockman J. Pediatric workforce: a look at pediatric endocrinology data from the American Board of Pediatrics. J Pediatr 2006: 149: 10-16.

17. Cho J-H, Chang S-A, Kwon H-S et al. Long-term effect of the internet-based glucose monitoring system on $\mathrm{HbAlc}$ reduction and glucose stability. Diabetes Care 2006: 29: 2625-2631.

18. Tamborlane WV. Fulfilling the promise of insulin pump therapy in childhood diabetes. Pediatr Diabetes 2006: 7 (Suppl. 4): 4-10. 